\title{
Dynamics Modeling of Transmission Gear Rattle and Factors Analysis Ya-Nan ZHAO ${ }^{2}$, Hong-Hui ZHANG ${ }^{1,2, a}$ \\ ${ }^{1}$ State Key Lab. of Vehicle NVH and Safety Technology, Chongqing, China \\ ${ }^{2}$ Key Lab. for Opto-electronic Technology \& Systems of Ministry of Education, Chongqing University, Chongqing, China \\ ahhzhang@cqu.edu.cn
}

Keywords: Transmission, Rattle noise, Dynamic model, Numerical analysis.

\begin{abstract}
Based on the vibration dynamics modeling for the single stage gear of transmission system, this paper is to understand the mechanism of transmission rattle. The dynamic model response using MATLAB and Runge-Kutta algorithm is analyzed, and the ways for reducing the rattle noise of the automotive transmission is summarized.
\end{abstract}

\section{Introduction}

As the increasing of requirement for automobile quality [1], the transmission rattle noise becomes more and more important for $\mathrm{NVH}$ performance. Generally, two factors is significant to the transmission rattle [2]: the external excitation caused by the input speed and torque from the engine, and the internal incentive factors caused by the time-varying stiffness, damping, gear side clearance and mesh error. This paper is trying to analyze the influencing factors for automotive transmission, and summarize the ways for rattle reduction.

\section{Dynamics Modeling of Stage Gear}

\section{Modeling of Single Stage Gear}

Based on the equivalent principle of inertia [3], rattle behavior of the gear system can be equivalent to the superposition of single gear pairs' vibration effect. For convenience, a single straight gear pair is used for the numerical modeling [4]. Dynamic model of single gear pair is shown in Fig. 1.

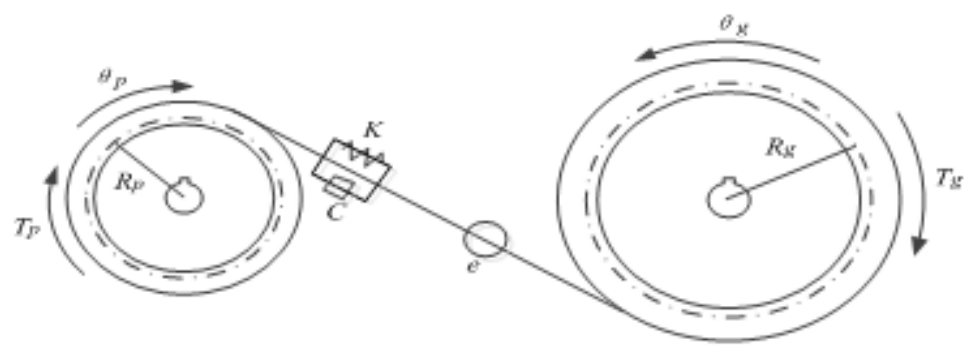

Fig.1. Dynamic model of single gear pair

The corresponding mathematical model is built under the second Newton's law. Considering the meshing error, the force analysis on the driving and driven gear is carried out. The equations are as followings:

$$
\begin{aligned}
& I_{p} \ddot{\theta}_{p}+R_{p} C\left(R_{p} \dot{\theta}_{p}-R_{g} \dot{\theta}_{g}-\dot{e}(t)\right)+ \\
& R_{p} K(t)\left(R_{p} \theta_{p}-R_{g} \theta_{\mathrm{g}}-e(t)\right)=T_{s}+T_{v}(t) \\
& I_{g} \ddot{\theta}_{g}-R_{g} C\left(R_{p} \dot{\theta}_{p}-R_{g} \dot{\theta}_{g}-\dot{e}(t)\right)- \\
& R_{g} K(t)\left(R_{p} \theta_{p}-R_{g} \theta_{\mathrm{g}}-\mathrm{e}(t)\right)=-T_{g}(t)
\end{aligned}
$$


Where $\mathrm{p}$ and $\mathrm{g}$ are the subscripts which demonstrating driving and driven gears. $I_{p}, I_{g}$ are the rotational inertia; $\theta_{p}$ and $\theta_{g}$ are the torsional vibration angular displacements; $R_{p}$ and $R_{g}$ is the base radius; $\mathrm{T}_{\mathrm{p}}$ and $\mathrm{T}_{\mathrm{g}}$ is the torque; $K, C$ is the torsional stiffness and damping coefficient respectively. $e(t)$ is the gear dynamic meshing error; $T_{s}, T_{v}(t)$ is the stable component and variation component of driving torque.

For the convenience, the relative vibration displacement between the two meshing teeth is [5]:

$$
x=R_{p} \theta_{p}-R_{g} \theta_{g}
$$

Considering the clearance, actual deformation and tooth surface vibration displacement are no longer equal [6]. Deformation happens only when the relative displacement is greater than the clearance. Set $\mathrm{b}$ as teeth clearance on both sides, the actual deformation can be expressed by function $f_{r}(t)$ :

$$
f_{r}(t)=\left\{\begin{array}{lc}
x-b, & x>b \\
0, & -b \leq x \leq b \\
x+b, & x<-b
\end{array}\right.
$$

Combining the Eqs (1-3) and dynamics equation is as Eq. 5: $m_{e} \ddot{x}(t)+C[\dot{x}-\dot{e}(t)]+K(t) f_{r}(x-e(t))=F_{T}-m_{e} \ddot{e}(t)(5)$

Where $m_{e}$ is the equivalent mass of gear pair, $F_{T}$ is the total load of transferring between teeth.

\section{Rattle Criterion of Gear Pair}

According to the theory of gear rattle threshold [7], whether rattle happens depends on the drive torque of the driven gear. When the driving torque is positive, meshing between the driving and driven gear is normal, rattle won't occur. When the driving torque is negative, the normal gearing is destroyed and then rattle occurs [8]. The relationship of driving torque, trailing torque, inertia torque is:

$$
T_{\text {drive }}=T_{\text {drag }}-\left|T_{\text {inertia }}\right|
$$

Where $T_{\text {drive }}$ is the driving torque of driven gear, $T_{\text {drag }}$ is the trailing torque, $T_{\text {inertia }}$ is inertia torque.

Contact force, vibration angular velocity and vibration angular acceleration can act as the criterion for rattle occurrence, but for intuitive results this paper takes the actual deformation as the criterion.

In normal gearing condition, the relative displacement between the driving and driven gear is greater than the clearance and deformation exists [9]. Otherwise, the driven gear may work between tooth clearance and knock the driving gear back and forth. The axial relative displacement between two teeth can be described as:

$$
\Delta x=R_{p} \theta_{p}-R_{g} \theta_{g}-e(t)=x(t)-e(t)
$$

Taking the deformation as rattle criterion:

$$
\left\{\begin{array}{c}
\Delta \bar{x}=|\Delta x|-b>0, \text { no rattle } \\
\Delta \bar{x}=|\Delta x|-b \leq 0, \text { rattle occurs }
\end{array}\right.
$$

\section{The Numerical Analysis for Rattle Occurrence}

\section{The Model Pre-processing}

In order to get more accurate result, the mesh stiffness data and error data in this paper are both given in the form of harmonic synthesis with $6^{\text {th }}$ order. The equations are as follows:

$$
K(t)=k_{m}+\sum_{i=1}^{6} k_{i} \cos \left(i \omega_{e} t+\phi_{i}\right)
$$




$$
e(t)=e_{m}+\sum_{j=1}^{6} e_{j} \cos \left(j \omega_{e} t+\phi_{j}\right)
$$

As the different magnitude of gear parameters such as stiffness and damping in the mode, using the dimensionless processing model is important for comparison. Considering the input torque variation component, the mathematical equation is described as follows:

$$
\begin{gathered}
\ddot{y}(t)+2 \xi \dot{y}(t)+\left[1-\sum_{i=1}^{6} \bar{k}_{i} \cos \left(i \omega t+\phi_{i}\right)\right] f[y(t)]= \\
F_{T}{ }^{\prime}+F_{V}{ }^{\prime} \cos (i \omega t)+\omega^{2} \sum_{j=1}^{6} m_{e}{ }^{2} \bar{e}_{j} \cos \left(j \omega t+\phi_{j}\right)
\end{gathered}
$$

Where $m_{e}=\frac{I_{p} I_{g}}{I_{p} R_{g}^{2}+I_{g} R_{p}^{2}}, \quad \xi=\frac{C}{2 \sqrt{k_{m} m_{e}}}, \omega=\frac{2 \pi z_{p} n_{p}}{60} \sqrt{\frac{m_{e}}{k_{m}}}, F_{T}^{\prime}=\frac{F_{T}}{b_{0} \sqrt{k_{m} m}}, \overline{k_{i}}=\frac{k_{i}}{k_{m}}, \overline{e_{i}}=\frac{e_{i}}{b_{0}}$, $b_{0}=10^{-6}, f[y(t)]=f_{r}(x-e(t)) \times b_{0}$

\section{Analysis of the Result}

Using the parameters of a certain type transmission gear, we can carry out the example analysis of the model by Runge-Kutta algorithm. The gear parameters are shown in Table 1.

\section{Gear Clearance}

Ignoring the influence of the clearance change under the time-varying stiffness, and keeping other parameters unchanged, set clearance $2 \mathrm{~b}$ as $4,20,40 \mathrm{um}$ respectively, We can get the dynamic system response respectively as shown in Fig. 2.

Table 1. The gear pair parameters

\begin{tabular}{|c|c|c|c|c|c|c|}
\hline & $\begin{array}{c}\text { Number } \\
\text { of teeth }\end{array}$ & $\begin{array}{c}\text { Rotary inertia } \\
(\mathrm{kg} \cdot \mathrm{mm} 2)\end{array}$ & Modulus & $\begin{array}{c}\text { Contact } \\
\text { ratio }\end{array}$ & $\begin{array}{c}\text { Tooth width } \\
(\mathrm{mm})\end{array}$ & $\begin{array}{c}\text { Clearance } \\
\text { 2b (um) }\end{array}$ \\
\cline { 1 - 4 } Driving gear(p) & 39 & 1206 & 2.5 & 1.7564 & 85 & 5 \\
\cline { 1 - 3 } Driven gear(g) & 20 & 681 & 2.56 & 5 \\
\hline
\end{tabular}
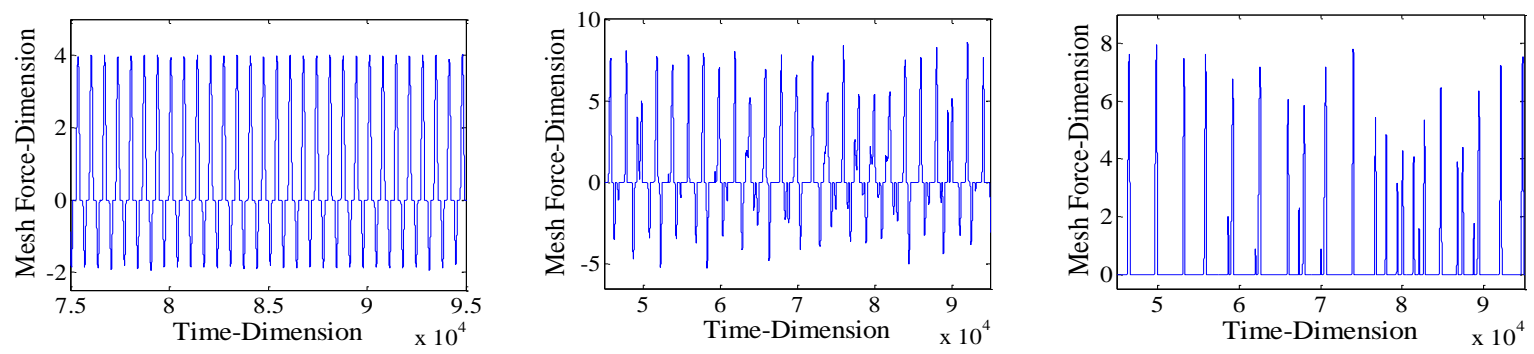

Fig. 2 (a). Meshing force amplitude changes as clearance increasing
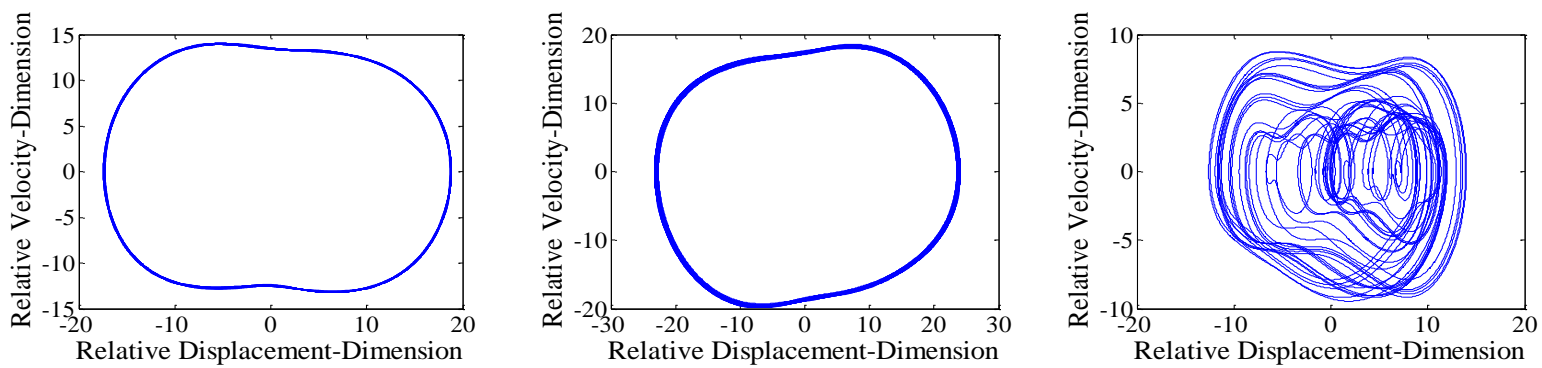

Fig. 2 (b). System phase diagram changes as clearance increasing 
As shown in the figures, with the increasing of clearance, rattle of gear pair varies from bilateral rap to unilateral tap within a certain range. System phase diagram shows that the original periodic response becoming chaos gradually as the increasing of clearance.

\section{Time-varying Stiffness Fluctuation}

Using the first harmonic component amplitude of time-varying stiffness as the research object, the influence on rattle response is analyzed under stiffness fluctuation. Setting k1 / km as $0.1,0.3,0.7$ respectively, and the dynamic response is shown in Fig. 3.
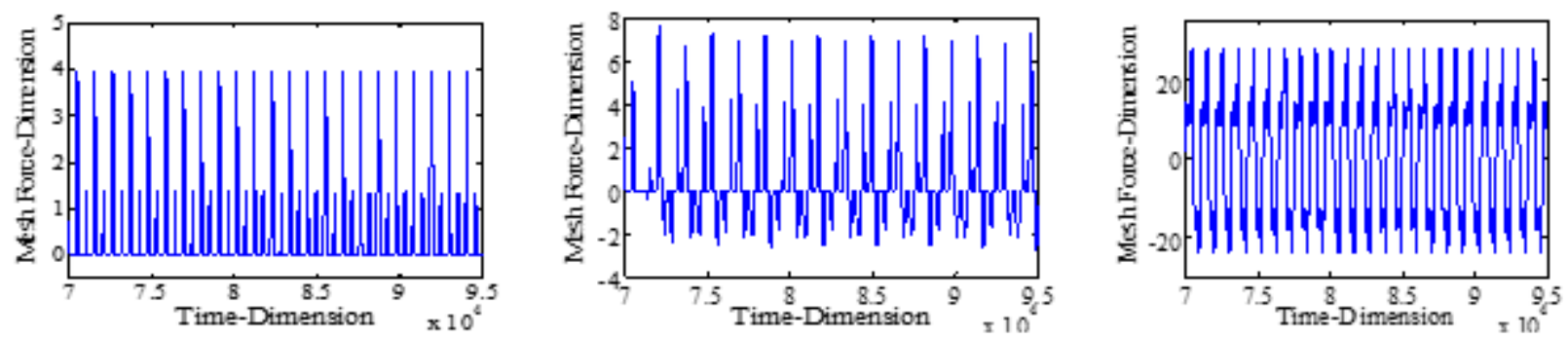

Fig.3 (a). Meshing force amplitude changes as stiffness fluctuation increasing
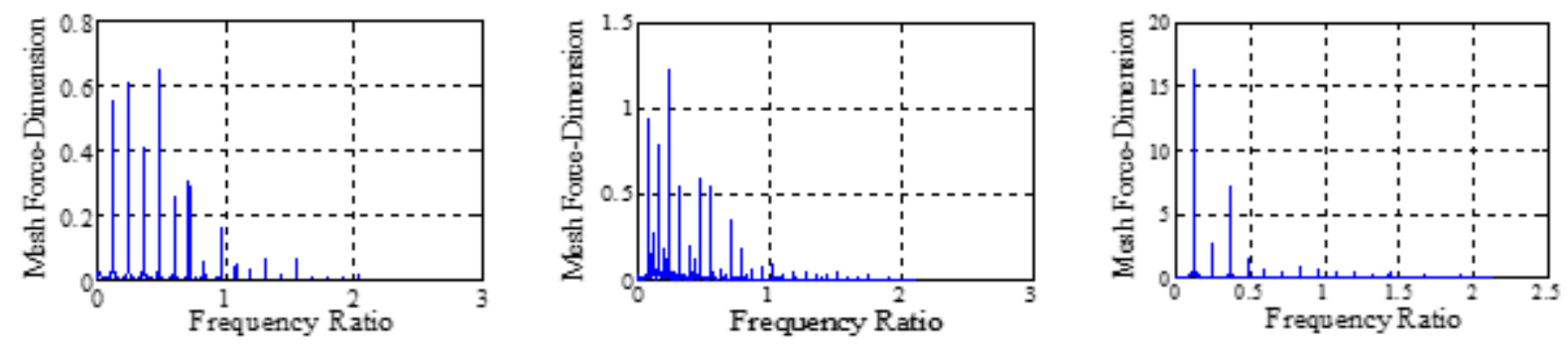

Fig. 3 (b). Meshing force frequency changes as stiffness fluctuation increasing
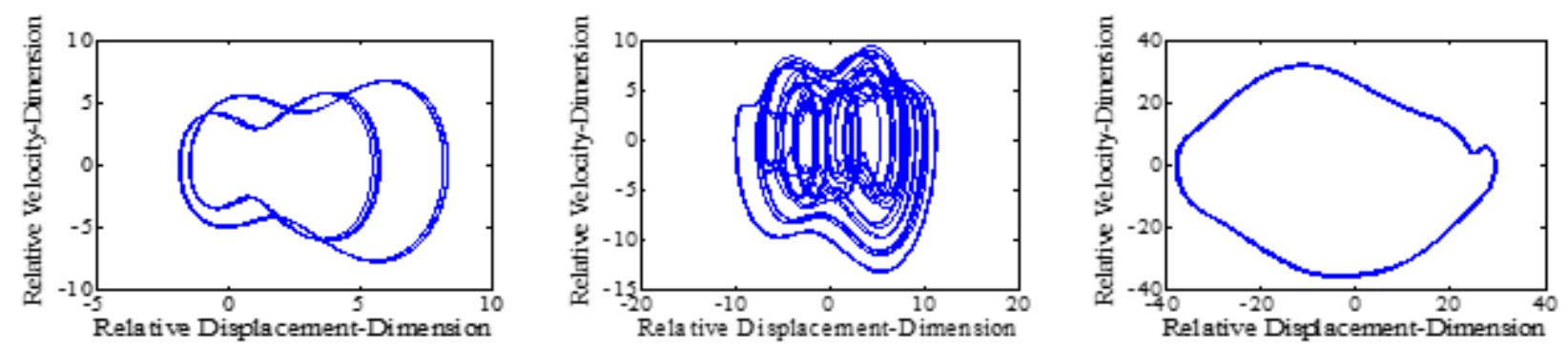

Fig. 3 (c). System phase diagram changes as stiffness fluctuation increasing

As shown in the figures, meshing force amplitude increases as the time-varying stiffness fluctuation component increasing. Rattle of gear pair varies from unilateral to bilateral within a certain range, the amplitude of frequency spectrum increases, and the main frequency gradually stands out.

\section{Meshing Damping}

The damping coefficient represents the combination of integration effects on the system. Set $\xi$ as 0.02, 0.05, 0.08 respectively, and the phase diagrams are shown in Fig. 4.

As shown in the figures, with the increasing of damping coefficient, the original chaotic response system is gradually becoming harmonic and periodic. It also proves that the appropriately increased damping effect can effectively improve the system stability and reduce the possibility of vibration rattle. 

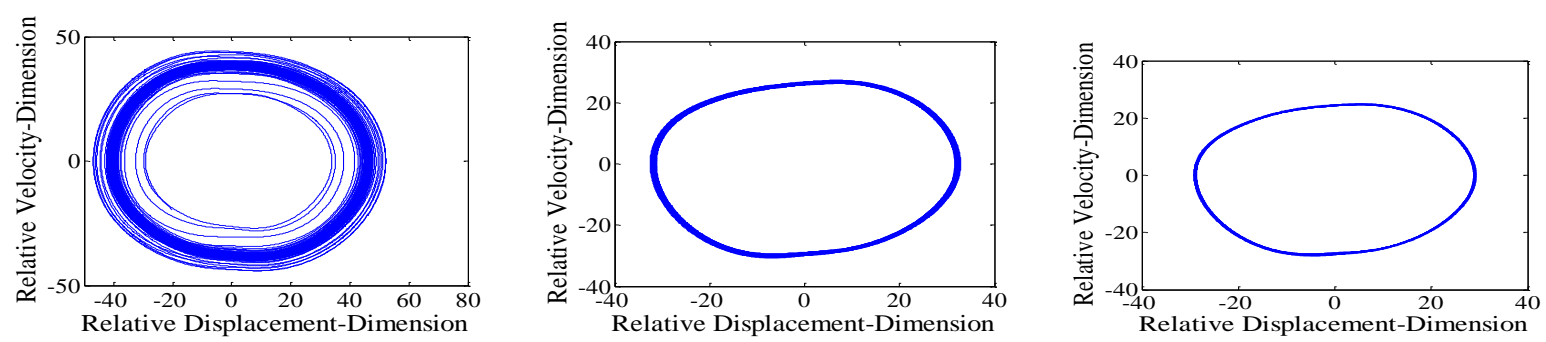

Fig. 4. System phase diagram changes as damping coefficient increasing

\section{Meshing Error Fluctuation}

In order to explore the incentive effect of the time-varying meshing error, we use the amplitude of the first harmonic component as the research object. Setting e1/em as $0.15 、 0.3 、 0.45$ respectively, the dynamic response is shown in Fig. 5.

As shown in the figures, the meshing force amplitude increases with the meshing error fluctuation somewhat. Unilateral rattle of gear pair varies to bilateral. The amplitude of frequency spectrum increases, and the main frequency gradually stands out.
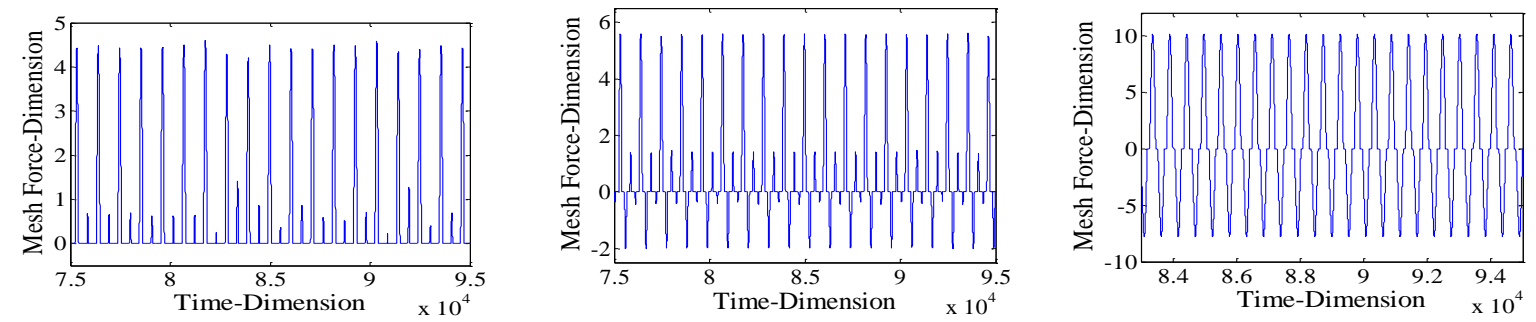

Fig. 5 (a). Meshing force amplitude changes as meshing error fluctuation increasing
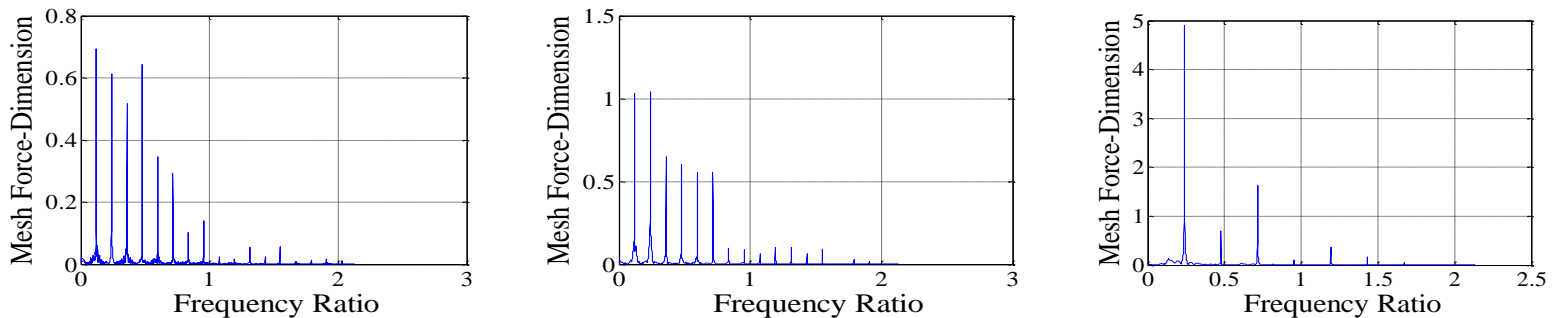

Fig. 5 (b). Meshing force frequency changes as meshing error fluctuation increasing

\section{Input Speed}

Input speed determines the meshing frequency of the system. To explore the relationship between the rattle and input speed, we set the wheel input speed $\mathrm{np}$ as 2000, 3000, $4000 \mathrm{r} / \mathrm{min}$, the response is shown in Fig. 6.

The meshing frequency comes close to natural frequency firstly, and then get far away again with the increasing input speed, and the system response is also changed from periodic to chaotic finally.
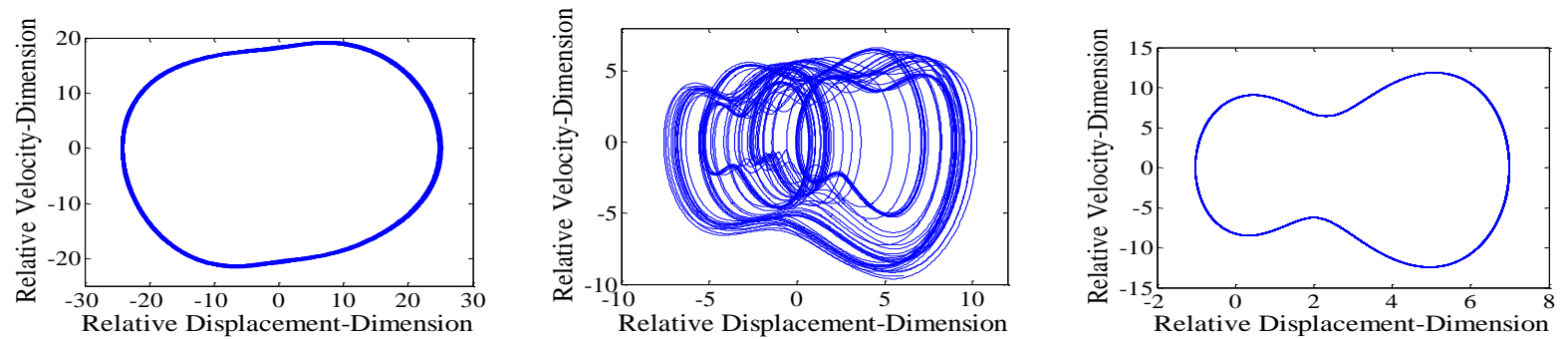

Fig. 6. System phase diagram changes as input speed increasing 


\section{Input Torque Fluctuation}

To explore the effect of torque fluctuation on the gear rattle, keeping other parameters unchanged, set FV/FT as 0.05, 0.2, 0.4 respectively, and the response is shown in Fig. 7.

Meshing force and frequency amplitude gradually increase with the increment of the torque fluctuation and the rattle of gear pair varies from unilateral to bilateral.
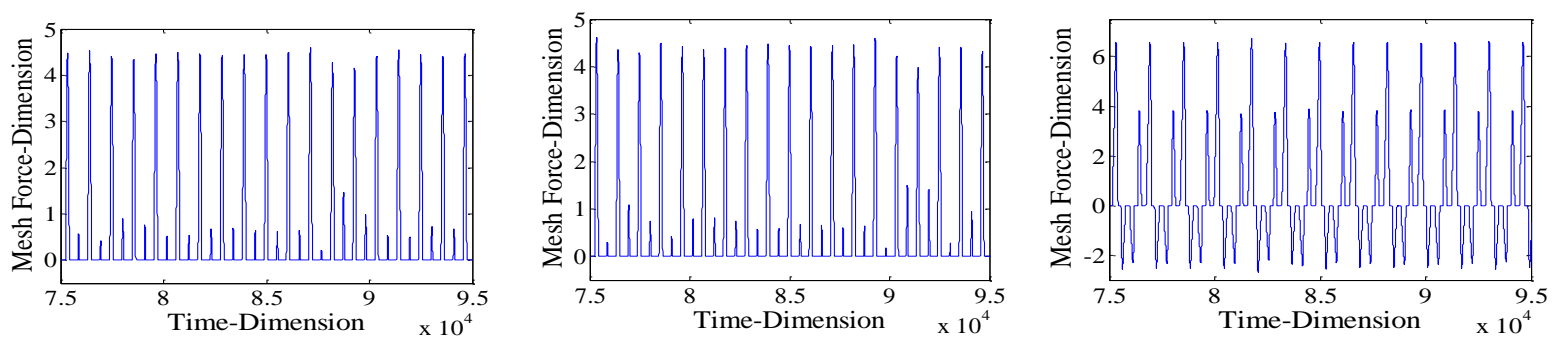

Fig. 7 (a). Meshing force amplitude changes as torque fluctuation increasing
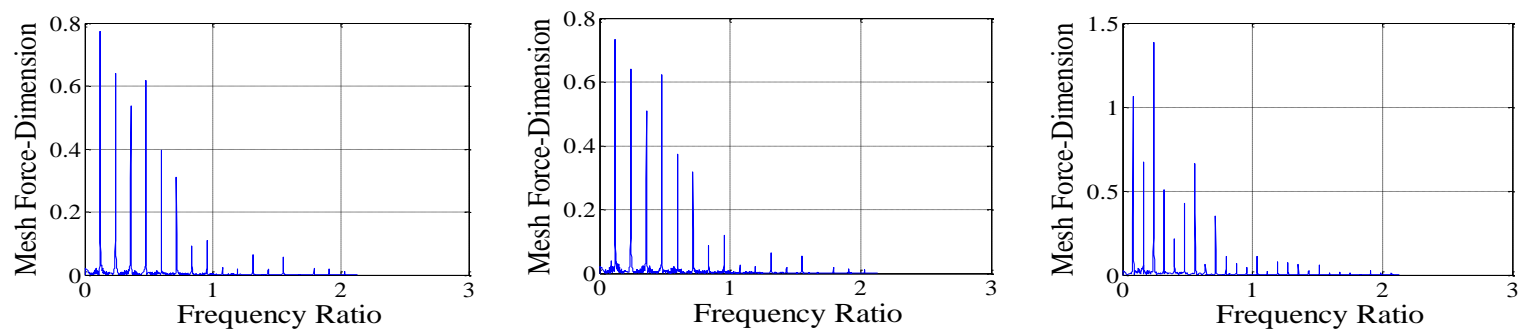

Fig. 7 (b). Meshing force frequency changes as torque fluctuation increasing

\section{Conclusions}

Based on the mechanism of transmission rattle and its influencing factors, a rattle dynamics mode of single gear pair is established and solution is implemented by Runge-Kutta algorithm. It shows that increased amplitude of gear clearance, time-varying stiffness fluctuation, meshing error fluctuation, input torque fluctuation will increase the gear rattle force, make the rattle varies between unilateral and bilateral, and affect the system periodicity. The increased gearing damping will make the dynamic response smooth, and reduce the rattle to some extent. Input speed determines the system meshing frequency and which influences the periodicity of the system obviously.

\section{Acknowledgement}

This work is supported by the Opening Foundation of State Key Laboratory of Vehicle NVH and Safety Technology (NVHSKL-201402).

\section{References}

1. R. Brancati, E. Rocca, S. Savino, F. Farroni. Analysis of gear rattle by means of a wavelet-based signal processing procedure[J]. Meccanica, 48(6): 1399-1413, (2013)

2. O. Tangasawi, S. Theodossiades, H. Rahnejat. Lightly loaded lubricated impacts: Idle gear rattle[J]. Journal of Sound and Vibration, 308(3-5): 418-430 (2007)

3. M. Barthod, B. Hayne, J. L. Tébec, J. C. Pin. Experimental study of gear rattle excited by a multiharmonic excitation[J]. Applied Acoustics, 68(9): 1003-1025 (2007)

4. N. H. Oliver Johnson. Diagnosis and objective evaluation of gear rattle[J]. SAE, 381-396 (1991) 
5. YANG Wen, CHEN Hong-jiang, HUANG Da-xing. Computer Dynamic Simulation of Gears in Gearbox[J]. Tractor \& Farm Transporter, 01: 20-21 (2008)

6. H. Optiz. Dynamic behavior of spur and helical gears[J]. SME Semi-International Symposium Papers, Gears., 9: 89-95 (1967)

7. Zhang Zhijun, Li Hongcheng, Yang Xianwu, Wang Lanjun, Li Yao, Gear Rattle Analysis of Dual-Clutch Transmission Based on Mulit-Stiffness Damper[C]. SAE-China Congress, Peking, China, 2013. 5 (2013)

8. WANG Lian-sheng, HAO Zhi-yong, ZHENG Kang, LIU Gong-wen. Simulation and experiment on transmission gear rattle considering drag torque [J]. Journal of Zhejiang University (Engineering Science), 05: 911-916 (2014)

9. Theoretical \& Experimental Research of Gear Rattle Noise of A Double Intermediate Shaft Manual Transmission[J]. Automobile Technology, 11:1-6(2014) 